Research Paper

\title{
Whole-Body Vibration Training Effect on Physical Performance and Obesity in Mice
}

\author{
Chi-Chang Huang1, ${ }^{\boxplus}$, Tzu-Ling Tseng1,\#, Wen-Ching Huang², Yi-Hsiu Chung 3 , Hsiao-Li Chuang,"\#, and \\ Jyh-Horng $\mathrm{Wu}^{5,}$ \\ 1. Graduate Institute of Sports Science, National Taiwan Sport University, Taoyuan 33301, Taiwan \\ 2. Graduate Institute of Athletics and Coaching Science, National Taiwan Sport University, Taoyuan 33301, Taiwan \\ 3. Molecular Imaging Center and Department of Nuclear Medicine, Chang Gung Memorial Hospital, Taoyuan 33305, Taiwan \\ 4. National Laboratory Animal Center, National Applied Research Laboratories, Taipei 11529, Taiwan \\ 5. Department of Forestry, National Chung Hsing University, Taichung 40227, Taiwan \\ \# These authors contributed equally to this work.
}

$\triangle$ Corresponding author: Dr. Chi-Chang Huang: Graduate Institute of Sports Science, National Taiwan Sport University, No. 250, Wenhua 1st Rd., Guishan Township, Taoyuan County 33301, Taiwan. Tel.: +886-3-3283201 (ext. 2619). Fax: +886-3-3280592. E-Mail: john5523@ntsu.edu.tw. Dr. Jyh-Horng Wu: Department of Forestry, National Chung Hsing University, No. 250, Kuo-kuang Rd., Taichung 40227, Taiwan. Tel.: +886-4-22840345 (ext. 136). Fax: +886-4-22851308. E-Mail: eric@nchu.edu.tw.

(c) Ivyspring International Publisher. This is an open-access article distributed under the terms of the Creative Commons License (http://creativecommons.org/ licenses/by-nc-nd/3.0/). Reproduction is permitted for personal, noncommercial use, provided that the article is in whole, unmodified, and properly cited.

Received: 2014.06.24; Accepted: 2014.09.14; Published: 2014.09.18

\begin{abstract}
The purpose of this study was to verify the beneficial effects of whole-body vibration (WBV) training on exercise performance, physical fatigue and obesity in mice with obesity induced by a high-fat diet (HFD). Male C57BL/6 mice were randomly divided into two groups: normal group $(n=6)$, fed standard diet (control), and experimental group $(n=18)$, fed a HFD. After 4-week induction, followed by 6 -week WBV of 5 days per week, the 18 obese mice were divided into 3 groups ( $n=6$ per group): HFD with sedentary control (HFD), HFD with WBV at relatively low-intensity $(5.6 \mathrm{~Hz}, 0.13 \mathrm{~g})(\mathrm{HFD}+\mathrm{VL})$ or high-intensity $(13 \mathrm{~Hz}, 0.68 \mathrm{~g})(\mathrm{HFD}+\mathrm{VH})$. A trend analysis revealed that WBV increased the grip strength in mice. WBV also dose-dependently decreased serum lactate, ammonia and CK levels and increased glucose level after the swimming test. WBV slightly decreased final body weight and dose-dependently decreased weights of epididymal, retroperitoneal and perirenal fat pads and fasting serum levels of alanine aminotransferase, CK, glucose, total cholesterol and triacylglycerol. Therefore, WBV could improve exercise performance and fatigue and prevent fat accumulation and obesity-associated biochemical alterations in obese mice. It may be an effective intervention for health promotion and prevention of HFD-induced obesity.
\end{abstract}

Key words: vibration training, exercise performance, fatigue, obesity, high fat diet

\section{Introduction}

The number of obese people is increasing rapidly, with an estimated 500 million obese adults in 2008 according to the World Health Organization [1]. Over the last decade, obesity has been increasingly recognized as a major health problem and is associated with heart disease, cardiovascular disease, type 2 diabetes, hypertension, reduced glucose tolerance, hypercholesterolemia and other chronic diseases.
Genetic, physiological and psychological factors, dietary habits, physical activity, lifestyle and social and environmental factors are responsible for the significant increase in prevalence of obesity and its sequels [2-4].

Exercise is an important strategy for weight loss in obese subjects and is followed by increasing energy expenditure. Moreover, regular exercise has a number 
of benefits such as improved cardiorespiratory fitness, reducing body weight [5] and improved quality of life [6]. Unfortunately, most obese people are not willing to maintain regular exercise.

Whole-body vibration (WBV), is a well-known light-resistance exercise based on automatic body adaptations to rapid and repeated oscillations of a vibrating platform [7]. WBV is increasingly being used in rehabilitation and fitness centers [8] as a non-invasive and non-pharmacological therapy for osteoporosis [9]. WBV can produce continuous eccentric-concentric muscular work with increased oxygen consumption [10]. Accordingly, WBV can positively affect body composition by reducing body fat accumulation and serum leptin [11, 12]. The other report also exhibited that WBV combined with endurance training could significantly increase resting energy expenditure for the improvement of body composition [13]. Vibration exercise was attracted a lot of attention as an exercise modality, which elevates metabolic rate and activates muscular adaption that could be a potential method for weight reduction [14]. The physical performance regarding to vibration exercise was focused on neuromuscular functions [15] or strength evaluation [16]. However, evidence for its effects is scarce, especially in obese subjects.

Because of limited reports of the effect of WBV on obesity-associated physical performance, biochemical profiles and body composition, we used a 4 -week-induced mouse model of obesity followed by a 6-week low- or high-frequency WBV training to investigate whether WBV improved physical performance and reduced obesity.

\section{Materials and methods}

\section{Animals and treatment}

Specific pathogen-free male C57BL/6 mice (4 weeks old) were purchased from BioLASCO (Yi-Lan, Taiwan). Animals were housed in the animal facility at National Taiwan Sport University (NTSU) at $22^{\circ} \mathrm{C}$, $50 \%$ to $60 \%$ relative humidity, with a 12 -h light-dark cycle (light on 7:00 AM). Distilled water and standard laboratory chow diet (No. 5001; PMI Nutrition International, Brentwood, MO, USA) were provided ad libitum. Before the experiments, the mice were acclimatized for 1 week to the environment and diet. The Institutional Animal Care and Use Committee (IACUC) of NTSU approved all animal experimental protocols, and the study conformed to the guidelines of the protocol IACUC-10214 approved by the IACUC ethics committee.

The experimental design was depicted as in Fig. 1. After 1-week acclimatization, 24 mice were divided randomly into two groups: the normal group $(n=6)$ was fed a standard chow diet (control) and the experimental group $(n=18)$ a high-fat diet (HFD). The 18 resulting obese mice were divided into three groups ( $n=6 /$ each group): 1) HFD with sedentary control (HFD), 2) HFD with relatively low-intensity WBV (HFD+VL; $5.6 \mathrm{~Hz}, 0.13 \mathrm{~g}$ peak acceleration and 2-mm vibration amplitude) or 3) HFD with relatively high-intensity WBV (HFD+VH; $13 \mathrm{~Hz}, 0.68 \mathrm{~g}$ peak acceleration and $2 \mathrm{~mm}$ vibration amplitude). Food intake and water consumption were recorded daily, and all animals were weighed weekly.

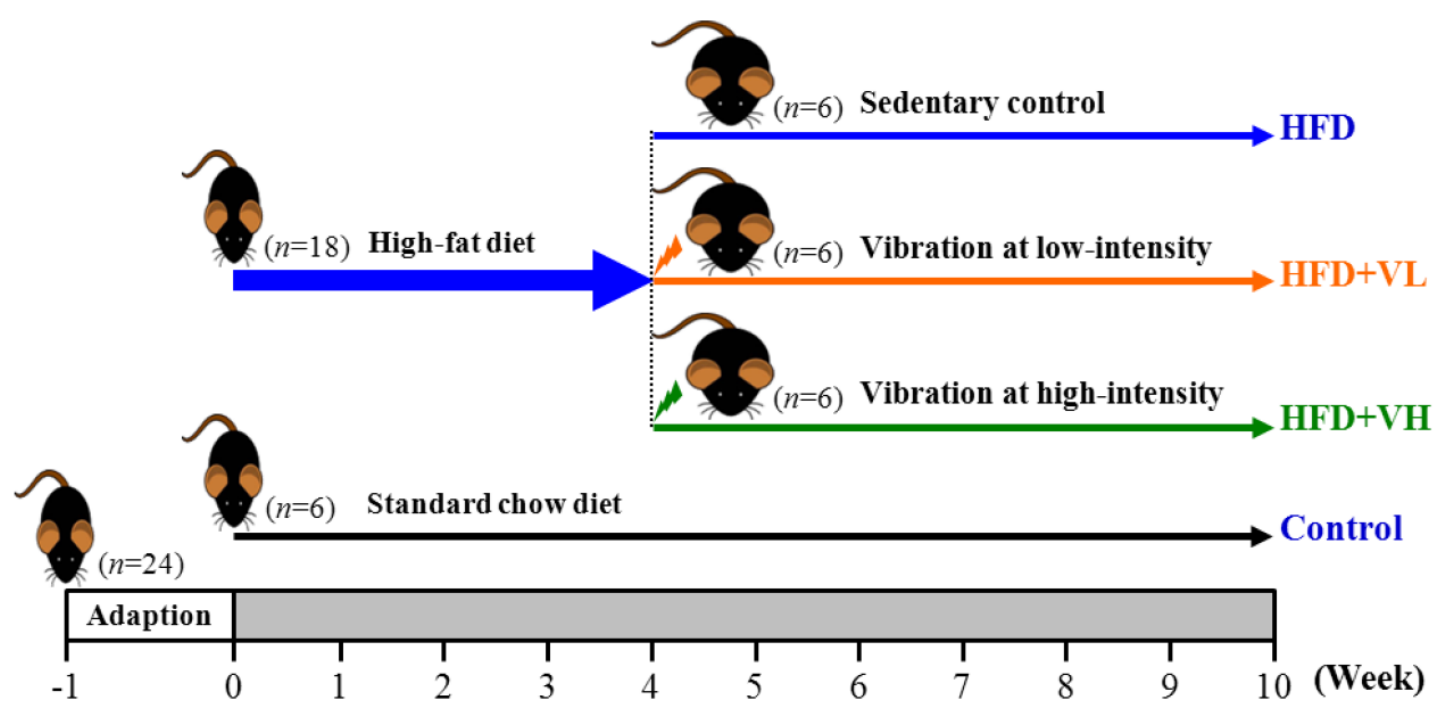

Figure I. Experimental design. After I-week adaption, 24 mice were divided randomly into two groups: the normal group was fed a standard chow diet (control, $\mathrm{n}=6$ ) and the experimental group with a high-fat diet (HFD, $n=18)$. After 4 weeks of the high-fat diet, the 18 obese mice were divided into three groups ( $n=6 /$ each group): I) HFD with sedentary control (HFD), 2) HFD with relatively low-intensity WBV (HFD+VL; $5.6 \mathrm{~Hz}, 0.13 \mathrm{~g}$ peak acceleration and 2-mm vibration amplitude) or 3) HFD with relatively high-intensity WBV (HFD+VH; $13 \mathrm{~Hz}, 0.68 \mathrm{~g}$ peak acceleration and $2 \mathrm{~mm}$ vibration amplitude). 


\section{HFD composition}

Mice were fed a standard chow diet or a HFD adapted from previous study [17] with some modification. The standard chow (No. 5001) contained 3.35 $\mathrm{kcal} / \mathrm{g}$ with $28.5 \%$ as protein, $13.4 \%$ as fat and $58.1 \%$ as carbohydrates. The HFD contained $8 \%(\mathrm{wt} / \mathrm{wt})$ soybean oil, $44 \%(\mathrm{wt} / \mathrm{wt})$ sweetened condensed milk (Original, Eagle Brand, Nestle) and $48 \%$ (wt/wt) standard chow, for $3.76 \mathrm{kcal} / \mathrm{g}$ with $15.5 \%$ as protein, $33.4 \%$ as fat and $51.1 \%$ as carbohydrates.

\section{WBV training protocol}

After 4-week obesity induction, HFD+VL and HFD+VH mice performed a WBV protocol [18] with some modifications. Briefly, mice were exposed to vibration on a vertically oscillating platform (BW-760, BodyGreen, Taipei). During vibration, mice were temporarily housed in 1 of 8 compartments of an acrylic cage fixed to the top of the platform. The vibration stimulus was applied for $15 \mathrm{~min}, 5 \mathrm{~d}$ / week for 6 weeks at a vibration frequency of 5.6 or $13 \mathrm{~Hz}$ and an acceleration magnitude of 0.13 or $0.68 \mathrm{~g}$. The vibration process was continuous for 15 minutes per day without interruption or rest. Control and HFD groups were placed in an identical cage for the same duration but did not receive any vibration stimulus.

\section{Forelimb grip strength}

A low-force testing system (Model-RX-5, Aikoh Engineering, Nagoya, Japan) was used to measure forelimb absolute grip strength as we previously described [19]; maximal force (grams) was recorded.

\section{Fatigue-associated biochemical indices}

After the 10-week experiment, mice underwent a 15-min swimming test without weight loading to evaluate fatigue-associated biochemical variables as in our previous studies [20-22]. Blood samples were immediately collected after the swimming exercise. Serum was collected by centrifugation at $1,500 \mathrm{~g}, 4^{\circ} \mathrm{C}$ for $10 \mathrm{~min}$. Lactate, ammonia, glucose and creatine kinase (CK) levels were determined by use of an auto-analyzer (Hitachi 7060, Hitachi, Tokyo).

\section{Blood biochemical assessments and histology of tissues}

At the end of the experiments, all mice were killed by $95 \% \mathrm{CO}_{2}$ asphyxiation, and blood was withdrawn by cardiac puncture after an 8 -h fast. Serum was collected by centrifugation, and levels of aspartate aminotransferase (AST), alanine aminotransferase (ALT), CK, glucose, total cholesterol (TC) and triacylglycerol (TG) were assessed by use of an auto-analyzer (Hitachi 7060).

The epididymal fat pad (EFP), retroperitoneal fat pad (RFP) and perirenal fat pad (PFP) from each group were photographed with use of a Cyber-shot (DSC-HX30V, Sony, Tokyo). The liver, muscle (gastrocnemius and soleus muscles in the back part of the lower legs) and fat pads were dissected, weighed and snap-frozen in liquid nitrogen before their storage at $-80^{\circ} \mathrm{C}$. Another set of liver tissues was removed intact and fixed in $10 \%$ neutral buffered formalin for $24 \mathrm{~h}$ before being processed for histopathologic analysis as we previously described [19]. Tissues were embedded in paraffin and cut into $4-\mu \mathrm{m}$ thick slices for morphological and pathological evaluation, then stained with hematoxylin and eosin (H\&E) and examined by use of a light microscope equipped with a CCD camera (BX-51, Olympus, Tokyo).

\section{Statistical analysis}

Data are expressed as mean \pm SEM. Statistical differences were analyzed by one-way ANOVA and the Cochran-Armitage test for trend analysis of dose-effect of WBV with SAS 9.0 (SAS Inst., Cary, NC, USA). $P<0.05$ was considered statistically significant.

\section{Results}

\section{Effect of 6-week WBV on body, water consumption and energy intake}

The initial body weight for control and HFD mice were similar (Fig. 2A). After 4-week induced obesity, the body weight was higher for HFD than control mice $(P<0.0001)$. After 6 -week WBV, the body weight was slightly but not significantly decreased with $\mathrm{WBV}+\mathrm{VH}$ than HFD alone $(P=0.0534)$. In all experiments, water consumption was lower for HFD, HFD+VL and HFD+VH than control mice $(P<0.05)$ (Fig. 2B), with no difference for HFD, HFD+VL and $\mathrm{HFD}+\mathrm{VH}$ mice $(P>0.05)$ (Fig. $2 \mathrm{~B}$ and $2 \mathrm{C})$. Additionally, energy intake did not differ among the 4 groups $(P>0.05)$ (Fig. 2B and 2C).

\section{Effect of 6-week WBV on forelimb grip strength}

The absolute grip strength of the mice was lower for HFD-alone than control mice (126 \pm 4 vs. $131 \pm 2 \mathrm{~g}$ ) (Fig. 3A). The grip strength was higher with HFD+VL and HFD+VH than HFD alone $(151 \pm 5$ and $164 \pm 5 \mathrm{~g}$ vs. $126 \pm 4 \mathrm{~g})(P<0.05)$. Therefore, HFD alone slightly lowered the absolute grip strength, and HFD+VL and $\mathrm{HFD}+\mathrm{VH}$ significantly increased relative absolute grip strength by 1.19 - and 1.30 -fold, respectively, as compared with HFD alone. Thus, WBV dose-dependently increased grip strength $(P<0.0001)$. The effect size of eta-squared is 0.74 as large significant strength. 
A

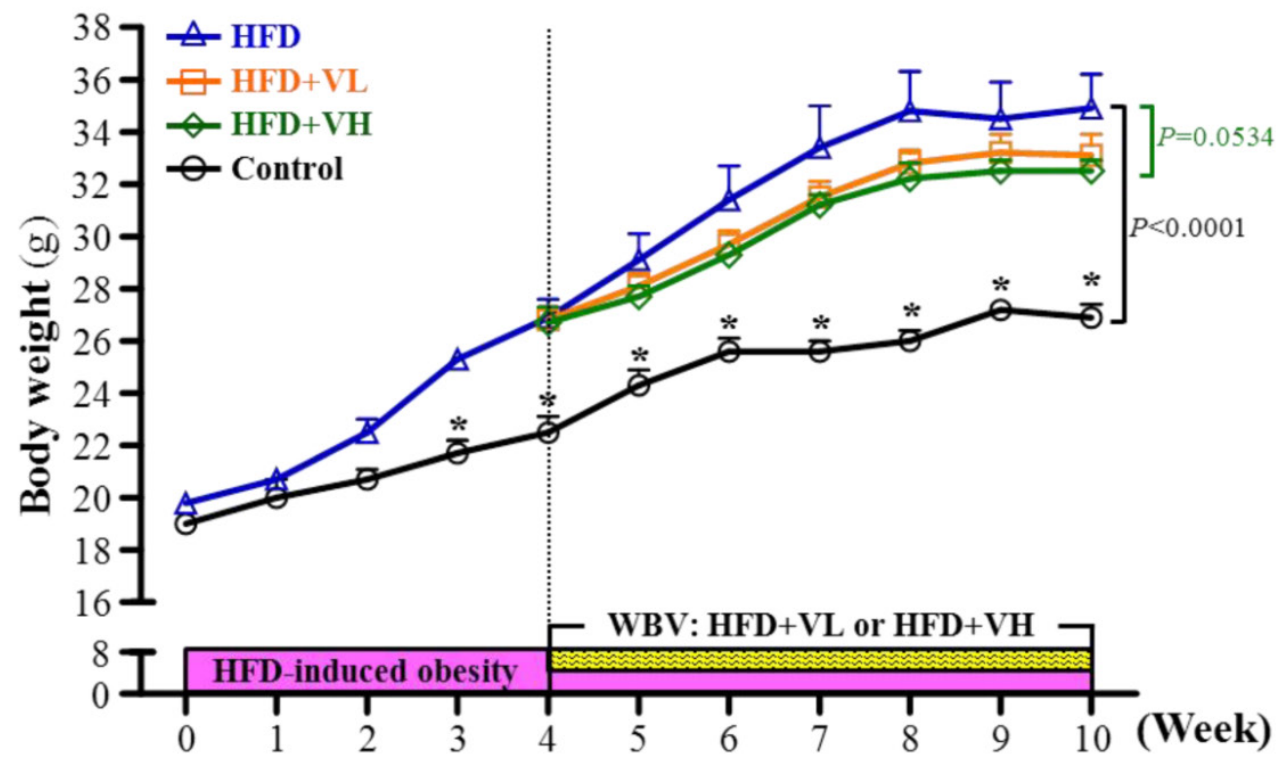

B

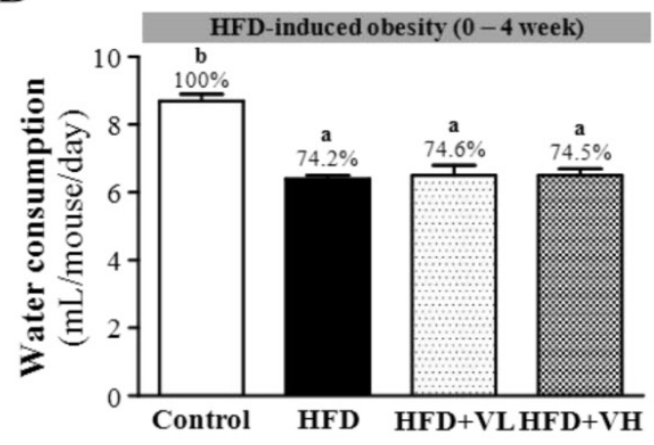

HFD-induced obesity ( $0-4$ week)

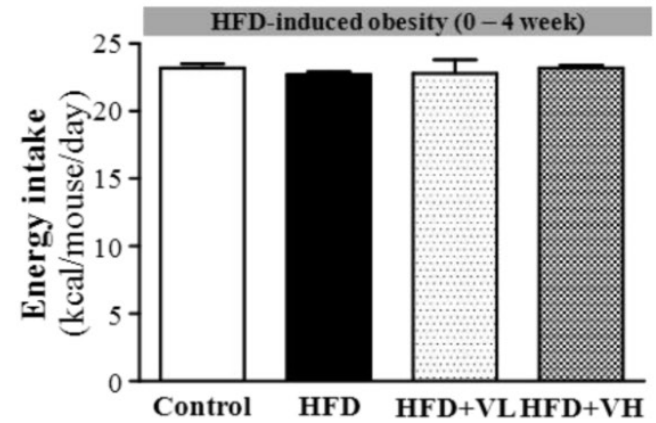

D

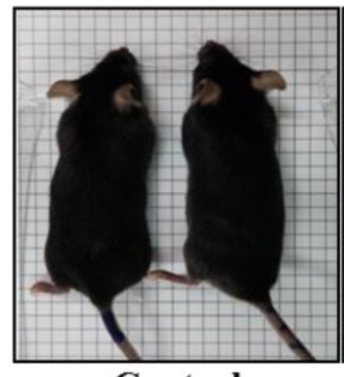

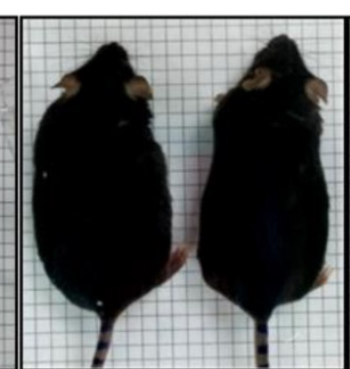

HFD
C
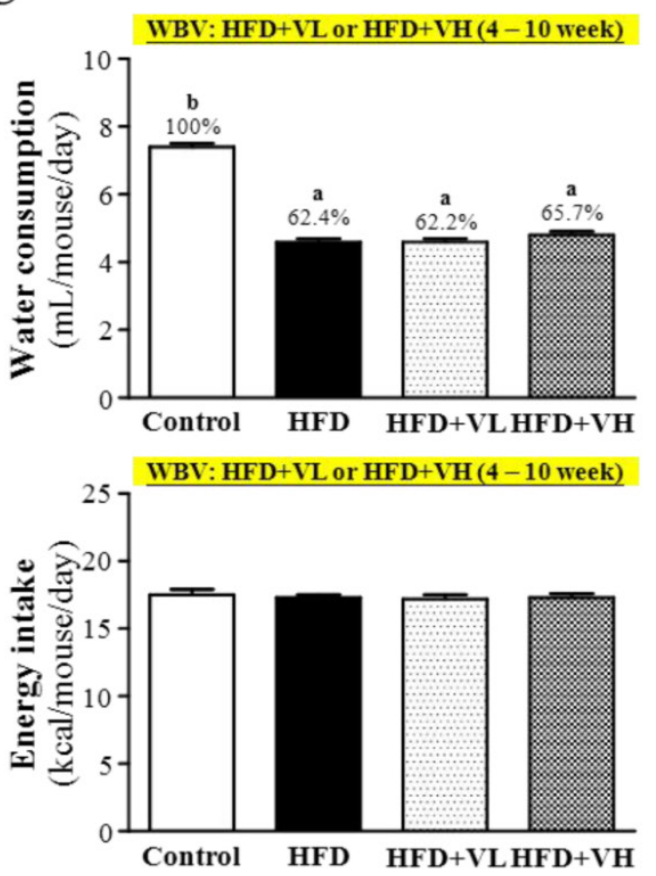

\section{Control}

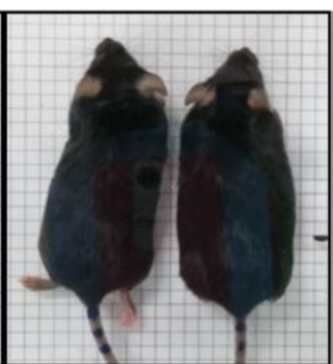

HFD+VL

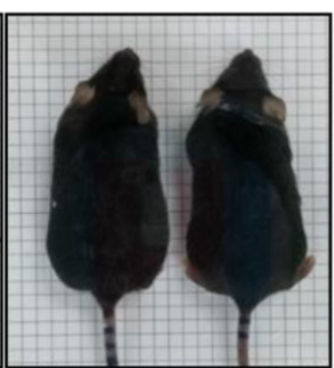

HFD+VH

Figure 2. Whole-body vibration training (WBV) prevented high-fat-diet-induced obesity in mice. Mice 5 weeks old were fed a HFD for up to 10 weeks combined with 6-week WBV at relative low intensity (HFD+VL) or high intensity $(\mathrm{HFD}+\mathrm{VH})$. Body weight $(\mathbf{A})$, water consumption and energy intake (B, C), and photographs $(\mathbf{B})$ of mice are shown. Data shown in A, B, C are the mean \pm SEM ( $n=6$ mice per group). One-way ANOVA was used for analysis. $* P<0.05$ compared with HFD group within each group. Different letters $(a, b)$ indicate significant difference at $P<0.05$. 
A

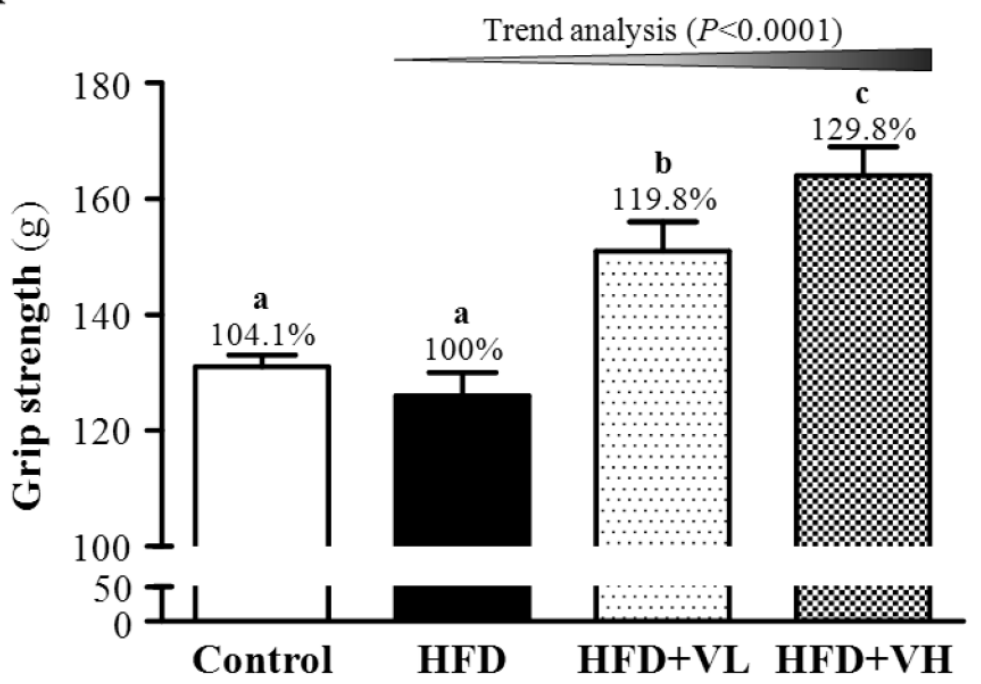

B

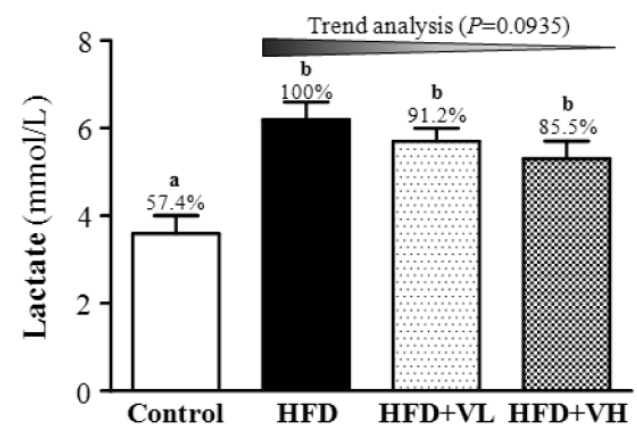

D

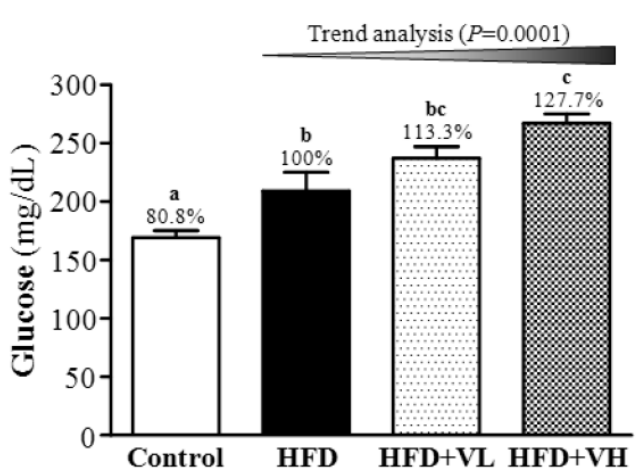

C

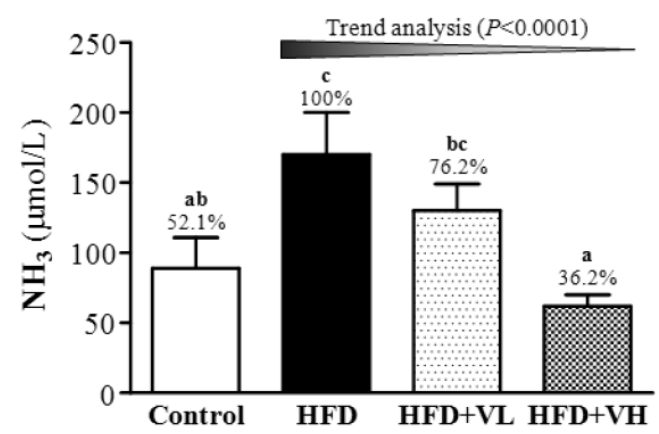

$\mathbf{E}$

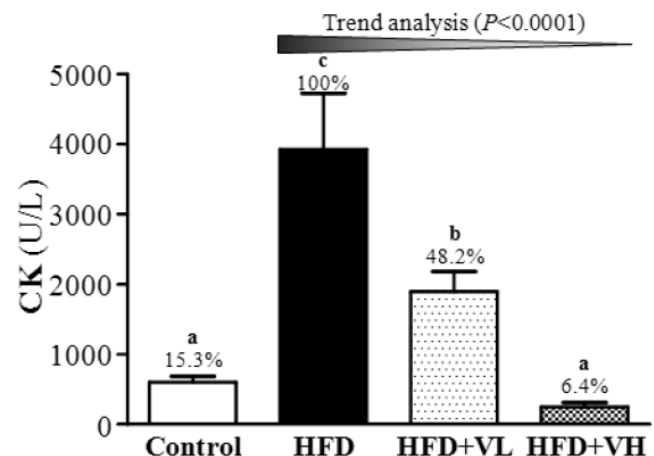

Figure 3. Effect of 6-week WBV on forelimb grip strength (A) and serum lactate (B), ammonia (C), glucose (D) and creatine kinase (CK) (E) levels after a I5-min swimming exercise challenge in HFD-induced obese mice. Data are mean \pm SEM ( $n=6$ mice/group). Different letters (a, b, $c$ ) indicate significant difference at $P<0.05$ by one-way ANOVA. The effect of vibration intensity was investigated by the Cochran-Armitage test for the dose-effect trend analysis. $* P<0.05$ was considered statistically significant.

\section{Effect of 6-week WBV on fatigue-associated biochemical indices}

After the 10-week experiment, mice underwent a 15-min swimming test to evaluate fatigue-associated biochemical variables (Fig. 3B-E). Serum levels of lactate, ammonia, glucose and CK were significantly higher in HFD-alone than control mice, by 1.74-, 1.92-, 1.24- and 6.54-fold, respectively. Serum levels of lactate, ammonia and CK were lower with HFD+VL or
HFD+VH than HFD alone, by $8.8 \%$ or $14.5 \%, 23.8 \%$ or 63.8 and $51.8 \%$ or $93.9 \%$, respectively, but the level of glucose was significantly increased with HFD+VL or HFD+VH than HFD alone, by 1.13- and 1.28-fold, respectively. Thus, WBV dose-dependently decreased serum lactate $(P=0.0935)$, ammonia $(P<0.0001)$ and CK $(P<0.0001)$ levels and increased glucose level $(P=0.0001)$. The effect size of eta-squared for lactate, ammonia, glucose and CK were $0.56,0.39,0.66$, and 0.66 , respectively. 


\section{Effect of 6-week WBV on fat pads}

EFP, RFP and PFP are white adipose tissues in the body. EFP, RFP and PFP mass was greater for HFD-alone than control mice, and WBV significantly decreased the mass (Fig. 4A). The weight of EFP, RFP and PFP was higher for HFD-alone than control mice by $77.0 \% \quad(P<0.05), \quad 84.9 \%$ $(P<0.05)$ and $93.1 \%(P<0.05)$, respectively (Fig. 4B). However, HFD+VL or HFD+VH could significantly decrease the weight as compared with HFD alone, by $22.2 \%$ or $29.8 \%, 43.9 \%$ or $60.5 \%$ and $17.5 \%$ or $18.0 \%$, respectively. WBV dose-dependently decreased the weight of EFP $(P=0.0281)$, RFP $(P<0.0001)$ and PFP $(P=0.0018)$. The effect size of eta-squared for EFP, RFP, and PFP were 0.76 , 0.68 , and 0.83 , respectively.

Figure 4. Effect of 6-week WBV on epididymal, retroperitoneal and perirenal fat pad mass (A), and visceral fat pad weight (B) in HFD-fed mice. Data are mean \pm SEM $(n=6$ mice per group). Different letters $(a, b, c)$ indicate significant difference at $P<0.05$. The effect of vibration intensity was investigated by the Cochran-Armitage test for the dose-effect trend analysis. $* P<0.05$ was considered statistically significant.
A
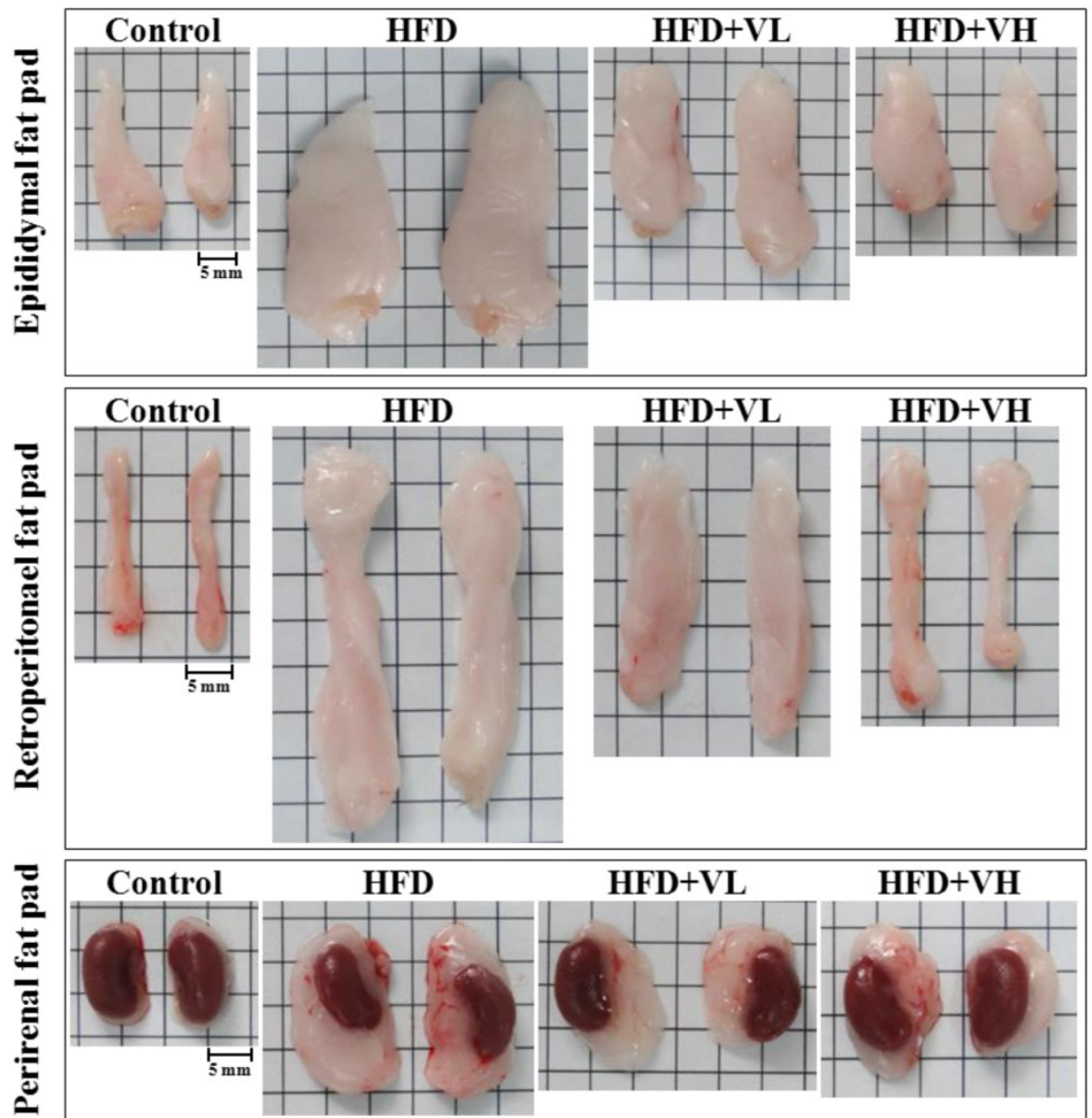

B
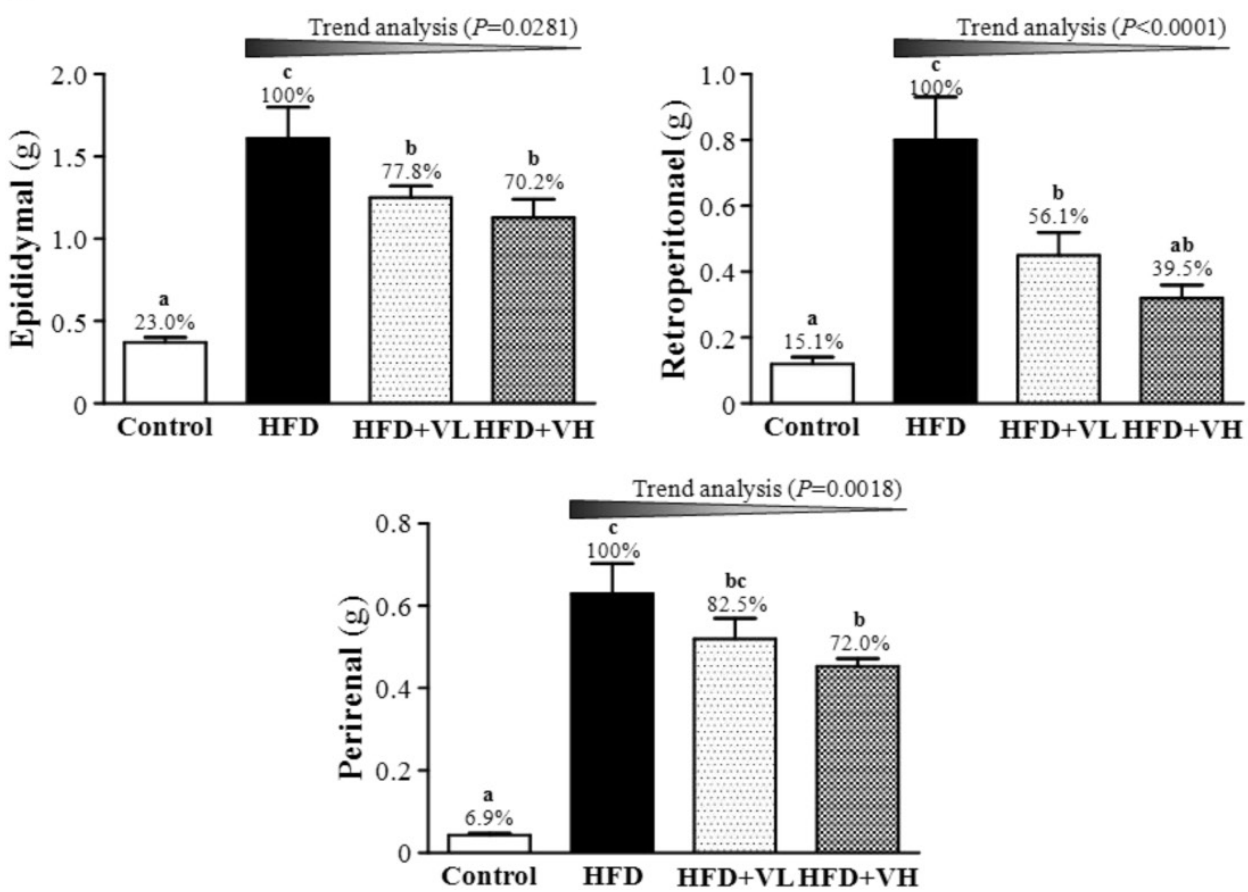
A

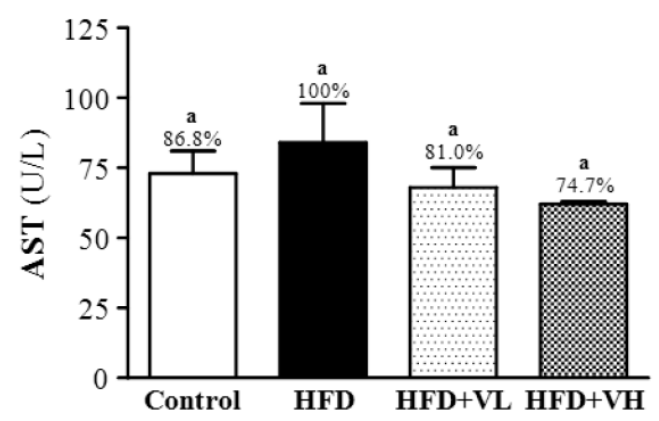

C

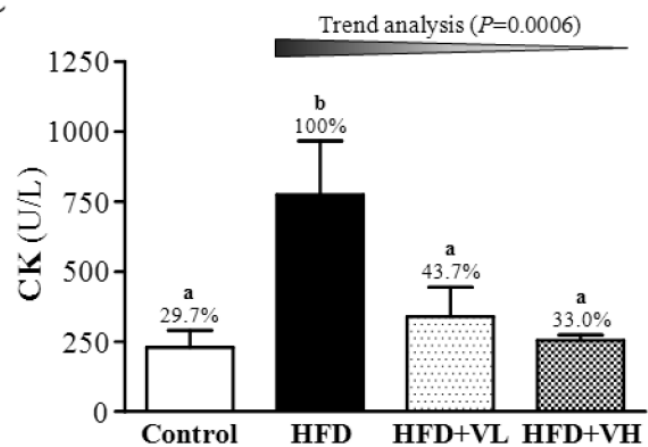

$\mathbf{E}$

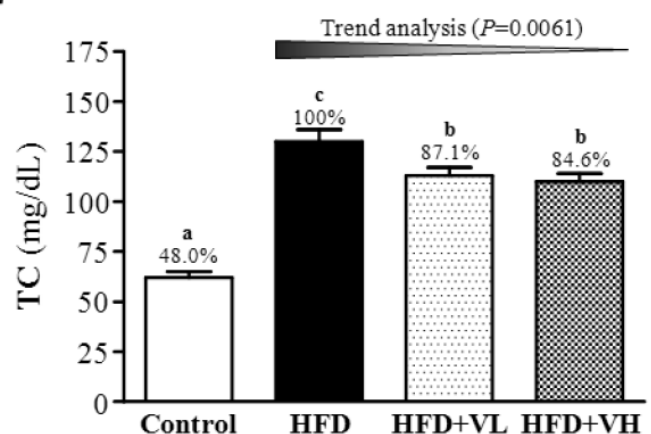

B

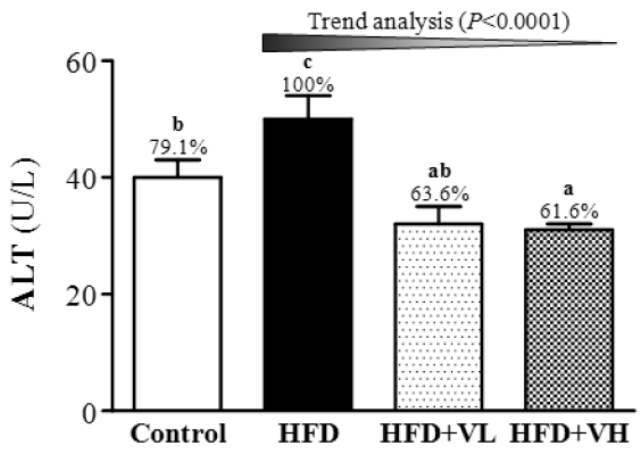

D

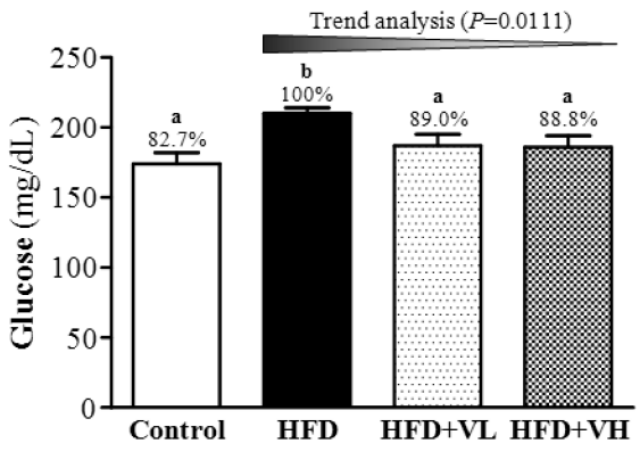

F

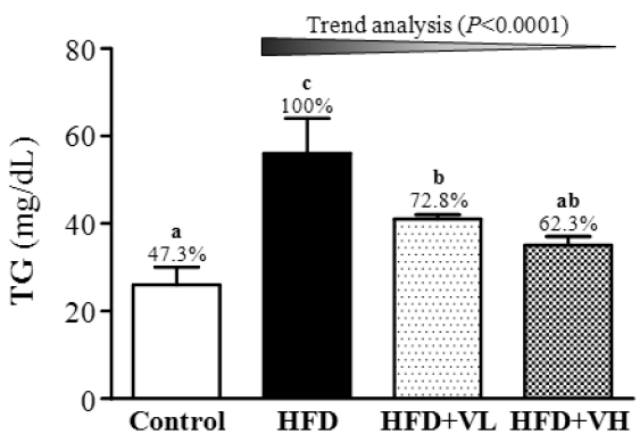

Figure 5. Effect of 6-week WBV on fasting serum levels of aminotransferase (AST) (A), alanine aminotransferase (ALT) (B), CK (C), glucose (D), total cholesterol (TC) (E) and triacylglycerol $(T G)(F)$ at the end of experiment in HFD-fed mice. Data are mean \pm SEM ( $n=6$ mice per group). Different letters (a, b, $c)$ indicate significant difference at $P<0.05$. The effect of vibration intensity was investigated by the Cochran-Armitage test for the dose-effect trend analysis. $* P<0.05$ was considered statistically significant.

\section{Effect of 6-week WBV on biochemical assessments}

As compared with control mice, HFD mice showed markedly increased serum levels of AST (1.15-fold, $\quad P<0.05)$, ALT $(1.26$-fold, $\quad P<0.05)$, CK (3.37-fold, $P<0.05)$, glucose (1.21-fold, $P<0.05)$, TC (2.08-fold, $P<0.05)$ and TG (2.11-fold, $P<0.05)$ (Fig. 5). Accordingly, WBV dose-dependently reduced ALT, $\mathrm{CK}$, glucose, TC and TG levels as compared with HFD alone $(P<0.0001, P=0.0006, P=0.0111, P=0.0061$ and $P<0.0001$, respectively). The levels of ALT, CK, glucose, TC, and TG were significantly lower with HFD+VL or HFD+VH than HFD alone, by $36.4 \%$ or $38.4 \%, 56.3 \%$ or $67.0 \%, 11.0 \%$ or $11.2 \%, 12.9 \%$ or $15.4 \%$ and $27.2 \%$ or $37.7 \%$, respectively. The effect size of eta-squared for ALT, CK, glucose, TC, and TG were $0.58,0.43,0.38,0.86$ and 0.52 , respectively.

\section{Effect of 6-week WBV on liver histology}

In H\&E staining of liver sections (Fig. 6), normal liver cells were arranged in proper order, with no lipid droplets (microvesicular fat) in lobes. However, HFD-alone mice showed an accumulation of lipid droplets in liver, whereas lipid droplets were rare in livers of HFD+VL and HFD+VH mice. Thus, obese mice with WVB for 6 weeks showed decreased accumulation of microvesicular fat and fatty liver. 

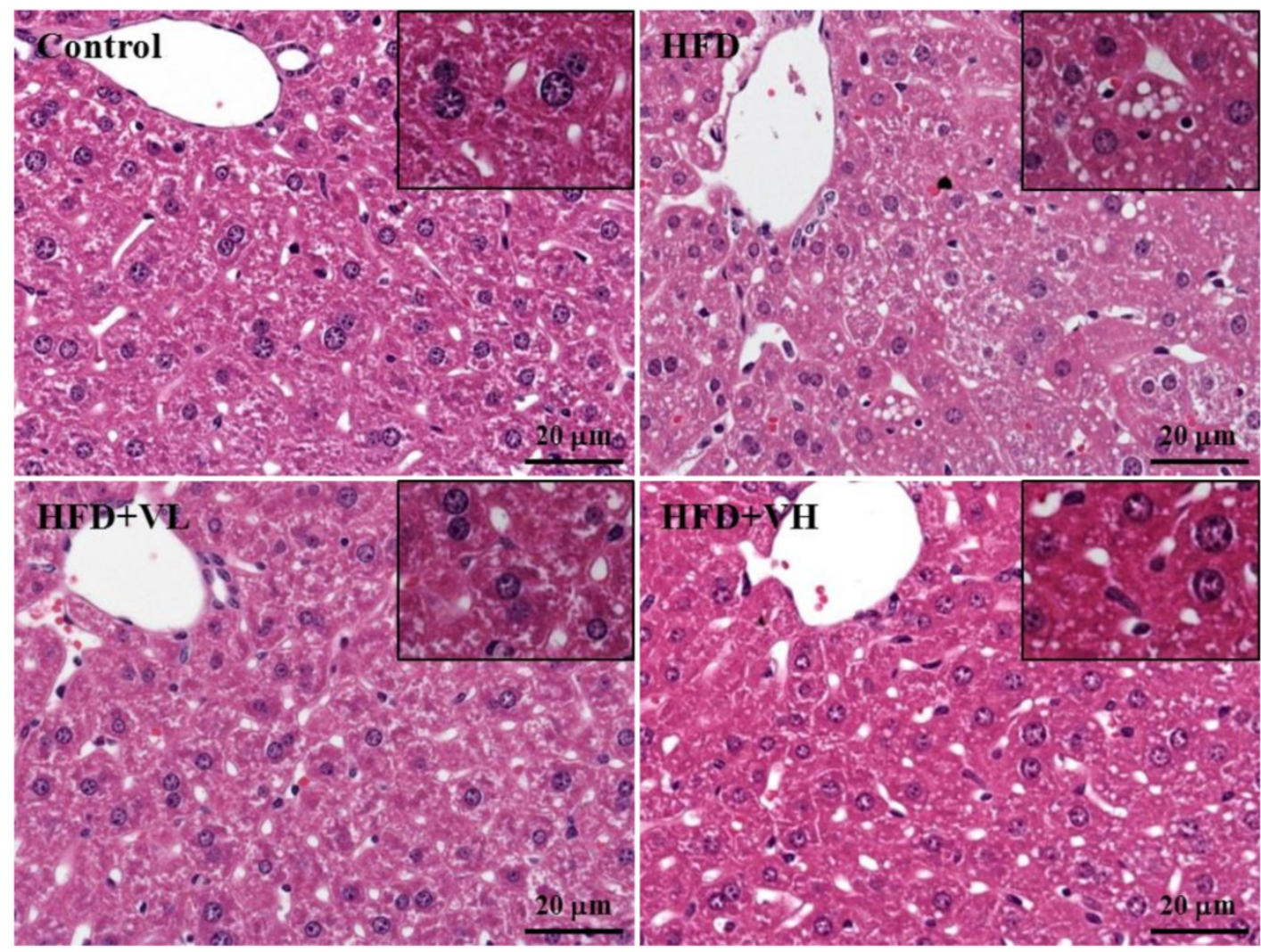

Figure 6. Effect of 6-week WBV on morphologic features of liver. Specimens were photographed by light microscopy. (H\&E staining, magnification: $\times 200$, Scale bar, $20 \mu$ m).

\section{Comment}

Obesity causes the accumulation of excess triglycerides in adipose tissue and leads to adverse health problems, including heart disease, cerebrovascular disease, diabetes, hypertension, hypercholesterolemia and other chronic diseases. It is becoming a major public health problem in developed and developing countries [1]. In recent years, vibration training, a systemic vibration sport, has been used to replace traditional resistance movement because it can increase muscle function, muscle strength and bone density and reduce excess body fat storage [11]. Previous studies have reported the benefit of WBV for patients with cystic fibrosis [23], multiple sclerosis [24] and stroke [25] but few have investigated its benefit for obesity. In this study, we established a mouse model of obesity with a high-calorie diet and used WBV training (low or high frequency) to investigate its efficacy on the formation of body fat in mice. The 6-week vibration training could significantly reduce the body weight of obese mice (Fig. 2A). The attenuated body weight may be due to the presence of a decreased amount of fatty tissue and a decreased adipocyte size (Fig. 4). Energy intake did not differ between control and HFD mice, but body weight was higher for HFD than control mice. We supposed that the HFD would decrease the basal metabolic rate and total daily energy expenditure as compared with controls and WBV would increase the basal metabolic rate and total daily energy expenditure. Thus, after 6-week WBV, body weight was slightly lower with relatively high-intensity WBV than HFD alone. Vissers et al. found that obese adults with WBV training and hypocaloric ( $\sim 600 \mathrm{kcal} /$ day) diet could reduce $8 \%$ of both body mass and body mass index [26]. Maddalozzo et al. showed that WBV led to approximately $10 \%$ less body weight, decreased proportion of body fat and lower level of serum leptin than in controls [11]. Recent studies also indicated that WBV could increase basal energy metabolism rate via greater oxygen uptake to values comparable to moderate walking $[10,27]$. Therefore, the body weight of our mice with WBV training may have been regulated by increased energy metabolism.

The forelimb grip strength test was developed decades ago and is a putative measure of muscular strength. Our previous study found that muscle strength was positively correlated with forelimb grip strength [22]. Maddalozzo et al. reported that vibration training could be used to replace traditional resistance movement to increase muscle function with similar resistance training effect [11]. Chen et al. also reported that eight weeks of vibration training improves jumping performance and body balance through neuromuscular adaptations [28]. In this 
study, we also found slightly lowered grip strength in HFD-alone than control mice, and WBV could increase the grip strength as compared with HFD alone.

After exercise, muscle fatigue is evaluated by important biochemical data, including lactate, ammonia, glucose and CK levels [29]. During high-intensity exercise, muscle obtains enough energy from anaerobic glycolysis, and abundant lactate is produced through glycolysis. The increasing lactate reduces the $\mathrm{pH}$ value, which can lead to various biochemical and physiological side effects on glycolysis, phosphofructokinase and muscular contractions [30]. In this study, after the 15-min swimming test, WBV could increase energy utilization (as blood glucose) in mice and decrease serum levels of lactate, ammonia and CK. Therefore, WBV could be a potential sport with an anti-fatigue effect.

White adipose tissues EFP, RFP and PFP are important indicators of obesity in the mouse body, and WBV could significantly decrease the formation of these adipose tissues (Fig. 4). WBV significantly reduced the body weight in obese mice, and the attenuated body weight may be due to a decreased EFP, RFP and PFP. Rubin et al. reported that low-intensity vibration reduced the differentiation of precursor cells to adipocytes, which suggests a possible mechanism of vibration training preventing fat accumulation [31]. Previous study also showed the vibration training could reduce fat mass but not lean mass $[11,12]$. WBV may positively affect body composition by decreasing the rate of fat accumulation while preserving lean mass.

Biochemical indicators in serum at the end of the experiment could provide health information about test animals. AST and ALT have been considered effective indicators of hepatic injury. WBV reduced AST and ALT in obese mice (Fig. 5). Obesity is always associated with the accumulation of excess lipid, impaired glucose tolerance and a high serum level of TG. Our H\&E staining of obese mouse livers revealed an accumulation of lipid droplets in the livers, and WBV could reduce the formation of lipid droplets (Fig. 6). HFD alone elevated TC and TG level as compared with controls, and WBV could significantly reduce the elevated levels of TC and TG in obese mice (Fig. 5E and $5 \mathrm{~F})$. DeAngelis et al. found that the accumulation of TC in liver parenchymal cells (fatty change or steatosis), a common liver pathology, is a well-established effect of obesity [32]. Rubin et al. demonstrated that WBV reduced $43 \%$ of TC level and $39 \%$ of free fatty acid level in livers [31]. In our study, HFD alone increased the level of glucose as compared with controls, whereas WBV decreased glucose level on fasting $8 \mathrm{~h}$ without intense exercise. Obesity is associated with insulin resistance [33, 34], thus WBV may improve the obesity-associated defect in insulin sensitivity. WBV dose-dependently reduced serum CK level in HFD mice as compared with HFD alone.

In conclusion, WBV may positively affect body weight, exercise performance, fatigue, fat accumulation and obesity-associated biochemical assessments in diet-induced obesity. It may be a potential sport for health promotion and have an anti-fatigue effect in preventing diet-induced obesity. For the future application with current results, the weight control, an important issue in modern society, could be intervened by diet management combined with vibration exercise, especially for obese population which couldn't tolerate the intensive aerobic exercise in the beginning. Therefore, the vibration exercise could be considered as one of exercise recipes for weight management and it also provided another exercise type for health population or athletes with effects of health promotion or anti-fatigue activity.

\section{Acknowledgments}

We thank Wen-Chyuan Chen for providing the vibration platform; Chien-Chao Chiu for conducting histological examination; Laura Smales (BioMedEditing, Toronto, Canada) for her careful reading of the manuscript; and financial support from the Ministry of Science and Technology of Taiwan, the successor to the National Science Council (grant no. NSC102-2628-H179-001-MY2).

\section{Competing Interests}

The authors have declared that no competing interest exists.

\section{References}

1. Chen HL, Tung YT, Tsai CL, Lai CW, Lai ZL, Tsai HC, Lin YL, Wang CH, Chen CM. Kefir improves fatty liver syndrome by inhibiting the lipogenesis pathway in leptin-deficient ob/ob knockout mice. Int J Obes (Lond). 2013; doi: 10.1038/ijo.2013.236

2. Grundy SM. Multifactorial causation of obesity: implications for prevention. Am J Clin Nutr. 1998; 67:563S-572S.

3. Hill JO, Peters JC. Environmental contributions to the obesity epidemic. Science. 1998; 280:1371-1374.

4. Wickelgren I. Obesity: how big a problem? Science. 1998; 280:1364-1367.

5. Wolfarth B, Rankinen T, Hagberg JM, Loos RJ, Pérusse L, Roth SM, Sarzynski MA, Bouchard C. Advances in exercise, fitness, and performance genomics in 2013. Med Sci Sports Exerc. 2014; 46:851-859.

6. Annesi JJ, Unruh JL. Relations of exercise, self-appraisal, mood changes and weight loss in obese women: testing propositions based on Baker and Brownell's (2000) model. Am J Med Sci. 2008; 335:198-204.

7. Coza A, Nigg BM, Dunn JF. Effects of vibrations on gastrocnemius medialis tissue oxygenation. Med Sci Sports Exerc. 2011; 43:509-515.

8. Bogaerts A, Delecluse C, Claessens AL, Coudyzer W, Boonen S, Verschueren $\mathrm{SM}$. Impact of whole-body vibration training versus fitness training on muscle strength and muscle mass in older men: a 1-year randomized controlled trial. J Gerontol A Biol Sci Med Sci. 2007; 62:630-635.

9. Von Stengel S, Kemmler W, Bebenek M, Engelke K, Kalender WA. Effects of whole-body vibration training on different devices on bone mineral density. Med Sci Sports Exerc. 2011; 43:1071-1079.

10. Rittweger J, Schiessl H, Felsenberg D. Oxygen uptake during whole-body vibration exercise: comparison with squatting as a slow voluntary movement. Eur J Appl Physiol. 2001; 86:169-173.

11. Maddalozzo GF, Iwaniec UT, Turner RT, Rosen CJ, Widrick JJ. Whole-body vibration slows the acquisition of fat in mature female rats. Int J Obes (Lond). $2008 ; 32: 1348-1354$ 
12. Milanese C, Piscitelli F, Zenti MG, Moghetti P, Sandri M, Zancanaro C. Ten-week whole-body vibration training improves body composition and muscle strength in obese women. Int J Med Sci. 2013; 10:307-311.

13. Wilms B, Frick J, Ernst B, Mueller R, Wirth B, Schultes B. Whole body vibration added to endurance training in obese women - a pilot study. Int J Sports Med. 2012; 33(9):740-743.

14. Cochrane DJ. Is vibration exercise a useful addition to a weight management program? Scand J Med Sci Sports. 2012; 22:705-771.

15. Perchthaler D, Grau S, Hein T. Evaluation of a six-week whole-body vibration intervention on neuromuscular performance in older adults. J Strength Cond Res. 2014; doi: 10.1519/JSC.0000000000000608.

16. Novotny SA, Mader TL, Greising AG, Lin AS, Guldberg RE, Warren GL, Lowe DA. Low intensity, high frequency vibration training to improve musculoskeletal function in a mouse model of duchenne muscular dystrophy. PLoS One. 2014; 9(8):e104339.

17. Levin BE, Dunn-Meynell AA. Reduced central leptin sensitivity in rats with diet-induced obesity. Am J Physiol Regul Integr Comp Physiol. 2002; 283:R941-948.

18. McKeehen JN, Novotny SA, Baltgalvis KA, Call JA, Nuckley DJ, Lowe DA. Adaptations of mouse skeletal muscle to low-intensity vibration training. Med Sci Sports Exerc. 2013; 45:1051-1059.

19. Chen WC, Huang WC, Chiu CC, Chang YK, Huang CC. Whey Protein improves exercise performance and biochemical profiles in trained mice. Med Sci Sports Exerc. 2014; 46:1517-1524.

20. Huang CC, Hsu MC, Huang WC, Yang HR, Hou CC. Triterpenoid-rich extract from Antrodia camphorata improves physical fatigue and exercise performance in mice. Evid Based Complement Alternat Med. 2012; 2012:364741.

21. Wang SY, Huang WC, Liu CC, Wang MF, Ho CS, Huang WP, Hou CC, Chuang HL, Huang CC. Pumpkin (Cucurbita moschata) fruit extract improves physical fatigue and exercise performance in mice. Molecules. 2012; 17:11864-11876.

22. Wu RE, Huang WC, Liao CC, Chang YK, Kan NW, Huang CC. Resveratrol protects against physical fatigue and improves exercise performance in mice. Molecules. 2013; 18:4689-4702.

23. Rietschel E, van KS, Fricke O, Semler O, Schoenau E. Whole body vibration: a new therapeutic approach to improve muscle function in cystic fibrosis? Int J Rehabil Res. 2008; 31:253-256.

24. Jackson KJ, Merriman HL, Vanderburgh PM, Brahler CJ. Acute effects of whole-body vibration on lower extremity muscle performance in persons with multiple sclerosis. J Neurol Phys Ther. 2008; 32:171-176.

25. van Nes IJ, Latour H, Schils F, Meijer R, van KA, Geurts AC. Long-term effects of 6-week whole-body vibration on balance recovery and activities of daily living in the postacute phase of stroke: a randomized, controlled trial. Stroke. 2006; 37:2331-2335.

26. Vissers D, Verrijken A, Mertens I, Van Gils C, Van de Sompel A, Truijen S, Van Gaal L. Effect of long-term whole body vibration training on visceral adipose tissue: a preliminary report. Obes Facts. 2010; 3:93-100.

27. Rittweger J, Ehrig J, Just $K$, Mutschelknauss M, Kirsch KA, Felsenberg D. Oxygen uptake in whole-body vibration exercise: influence of vibration frequency, amplitude, and external load. Int J Sports Med. 2002; 23:428-432.

28. Chen $\mathrm{CH}$, Liu C, Chuang LR, Chung PH, Shiang TY. Chronic effects of whole-body vibration on jumping performance and body balance using different frequencies and amplitudes with identical acceleration load. J Sci Med Sport. 2014; 17:107-112.

29. Brancaccio P, Maffulli N, Limongelli FM. Creatine kinase monitoring in sport medicine. Br Med Bull. 2007; 81-82:209-230.

30. Cairns SP. Lactic acid and exercise performance: Culprit or friend? Sports Med. 2006; 36:279-291.

31. Rubin CT, Capilla E, Luu YK, Busa B, Crawford H, Nolan DJ, Mittal V, Rosen CJ, Pessin JE, Judex S. Adipogenesis is inhibited by brief, daily exposure to high-frequency, extremely low-magnitude mechanical signals. Proc Natl Acad Sci USA. 2007; 104:17879-17884.

32. DeAngelis RA, Markiewski MM, Taub R, Lambris JD. A high-fat diet impairs liver regeneration in C57BL/6 mice through overexpression of the NF-kappaB inhibitor, IkappaBalpha. Hepatology. 2005; 42:1148-1157.

33. Olefsky J, Farquhar JW, Reaven G. Relationship between fasting plasma insulin level and resistance to insulin-mediated glucose uptake in normal and diabetic subjects. Diabetes. 1973; 22:507-513.

34. Beard JC, Ward WK, Halter JB, Wallum BJ, Porte D Jr. Relationship of islet function to insulin action in human obesity. J Clin Endocrinol Metab. 1987; 65:59-64. 